\title{
TRANSTIBIAL AMPUTEE GAIT WITH THE PRO-FLEX FOOT DURING LEVEL, DECLINE, AND INCLINE WALKING
}

\author{
Matthew Tomkin ${ }^{1,2 *}$, Hossein Gholizadeh ${ }^{1,3}$, Emily Sinitski ${ }^{1,4}$, Edward D Lemaire ${ }^{1,5}$ \\ ${ }^{1}$ Ottawa Hospital Research Institute, Centre for Rehabilitation Research and Development, Canada. \\ ${ }^{2}$ Ottawa-Carleton Institute for Biomedical Engineering, University of Ottawa, Canada. \\ ${ }^{3}$ Prosthetics \& Orthotics, The Ottawa Hospital Rehabilitation Centre, Canada. \\ ${ }^{4}$ Canadian Forces Health Services, Canada. \\ ${ }^{5}$ Faculty of Medicine, University of Ottawa, Canada. \\ *Email: mtomk062@uottawa.ca
}

DOI: https://doi.org/10.33137/cpoj.v1i2.32003

\section{INTRODUCTION}

The Össur Pro-Flex is a 3-blade carbon fiber prosthetic foot designed to provide greater ankle range of motion compared to conventional energy storing and returning prosthetic feet ${ }^{1,2}$. To provide knowledge on Pro-Flex biomechanical performance, kinematic and kinetic gait parameters were evaluated and compared with the Össur Pro-Flex XC prosthetic foot. Outcomes from this study provide clinical professionals with important decision-making knowledge about two innovative energy storing and returning prosthetic feet.

\section{METHODS}

Three K3 unilateral transtibial amputees were fitted with the Pro-Flex and the Össur Unity vacuum suspension system. All participants were previously fitted with the Pro-Flex XC and Unity system. Participants completed a warm-up trial and multiple self-paced walking trials in a virtual park (CAREN-extended virtual reality system) ${ }^{3}$. $3 \mathrm{D}$ motion was evaluated on level, $7^{\circ}$ decline, and $7^{\circ}$ incline walking conditions.

\section{RESULTS}

Pro-Flex ankle range of motion increased compared to the Pro-Flex XC during all conditions. Ankle push-off power was greater with the Pro-Flex XC during level and incline walking; however, similar power was observed during decline walking.

Table 1. Mean and standard deviation for ankle range of motion $\left({ }^{\circ}\right)$.

\begin{tabular}{|l|c|c|c|}
\multicolumn{1}{c|}{ Foot } & Level & Decline & Incline \\
\hline Pro-Flex & $22.85 \pm 2.63$ & $23.39 \pm 1.48$ & $20.01 \pm 3.31$ \\
\hline Intact & $30.71 \pm 11.04$ & $30.80 \pm 2.70$ & $37.01 \pm 12.48$ \\
\hline Pro-Flex XC & $18.75 \pm 4.29$ & $17.41 \pm 3.10$ & $14.88 \pm 2.94$ \\
\hline Intact & $28.97 \pm 7.42$ & $29.00 \pm 2.13$ & $34.60 \pm 10.00$
\end{tabular}

Table 2. Mean and standard deviation for ankle push-off power $(\mathrm{W} / \mathrm{kg})$.

\begin{tabular}{|l|c|c|c|}
\hline \multicolumn{1}{|c|}{ Foot } & Level & Decline & Incline \\
\hline Pro-Flex & $2.39 \pm 0.65$ & $2.27 \pm 0.32$ & $2.14 \pm 0.86$ \\
\hline Intact & $2.97 \pm 1.14$ & $3.00 \pm 0.87$ & $3.95 \pm 2.23$ \\
\hline Pro-Flex XC & $2.49 \pm 0.77$ & $2.27 \pm 0.81$ & $2.40 \pm 0.88$ \\
\hline Intact & $2.71 \pm 0.67$ & $2.59 \pm 0.86$ & $3.96 \pm 1.52$ \\
\hline
\end{tabular}

\section{CONCLUSION}

Pro-Flex ankle range of motion was greater than Pro-Flex $\mathrm{XC}$; however, Pro-Flex range of motion was smaller than reported by Heitzmann et al. ${ }^{1}$, perhaps due to differences in methodologies and study populations (e.g., Heitzmann had K3 and K4 participants). As expected, Pro-Flex peak ankle power generation did not surpass Pro-Flex XC during any of the conditions, since Pro-Flex XC was designed to generate appropriate power over a larger variety of activities and impact levels compared to ProFlex ${ }^{4}$.

\section{SIGNIFICANCE}

Pro-Flex provided greater ankle range of motion during level and slope self-paced walking, which may reduce compensatory gait strategies on sloped surfaces. However, the K3 participants may not have taken full advantage of Pro-Flex's ankle range of motion potential. While push-off powers were similar between feet, Pro-Flex XC was designed for higher activity and achieved greater energy return. Further research is required to identify when people benefit from greater ankle range of motion while maintaining ankle push-off power. Future studies with high activity transtibial and transfemoral amputees will provide a broader understanding about Pro-Flex's biomechanical performance. 
Level

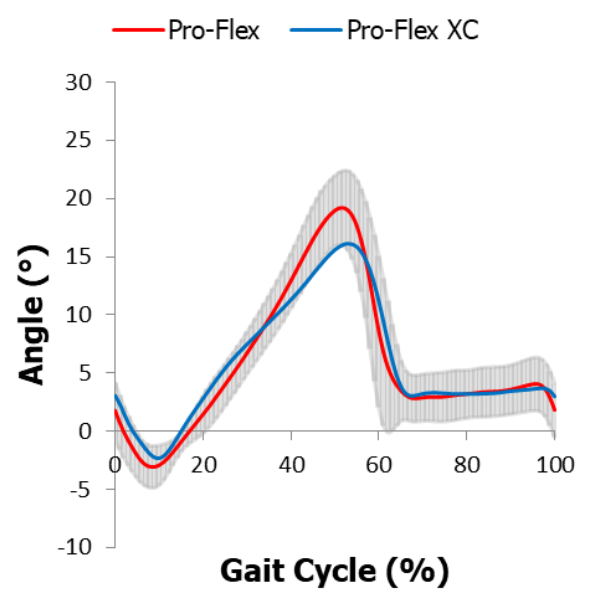

Level

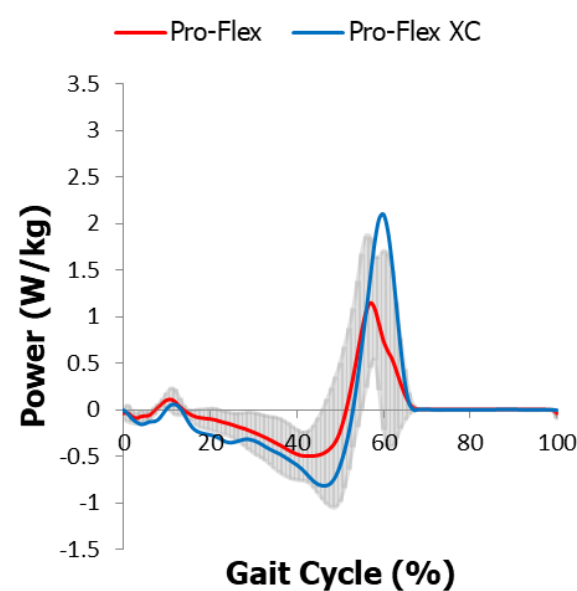

Decline

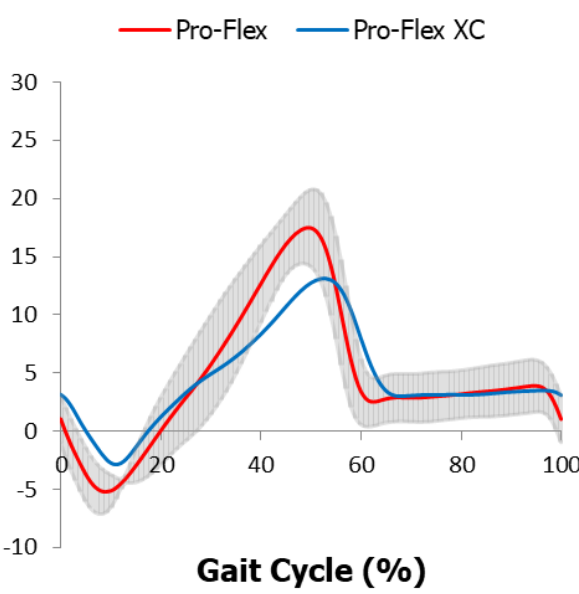

Decline

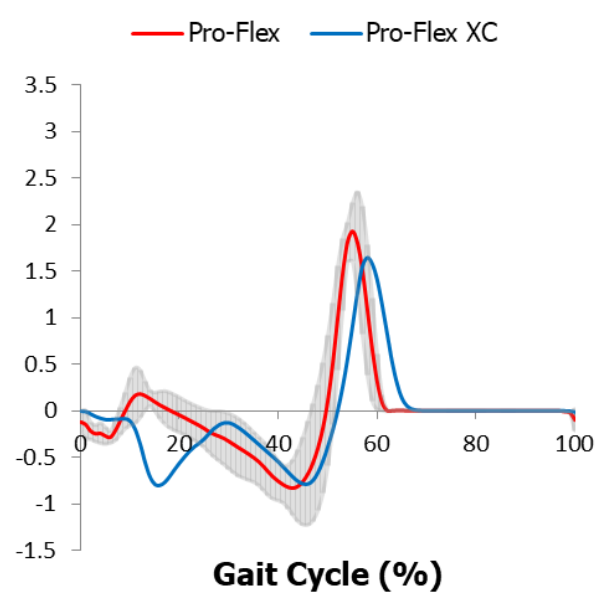

Incline

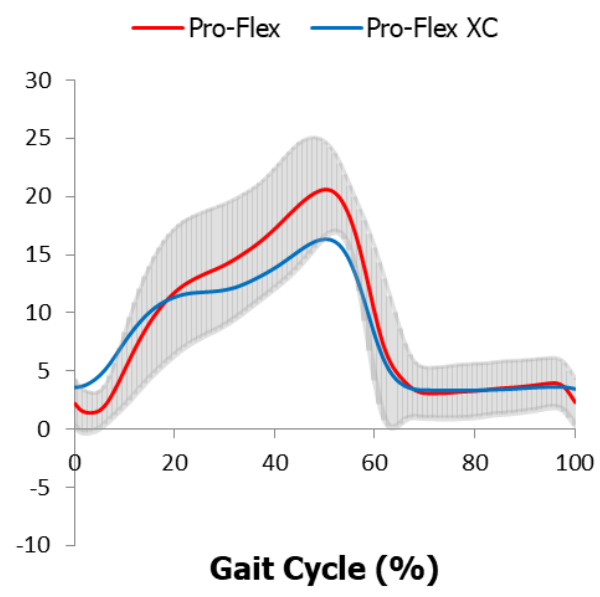

Incline

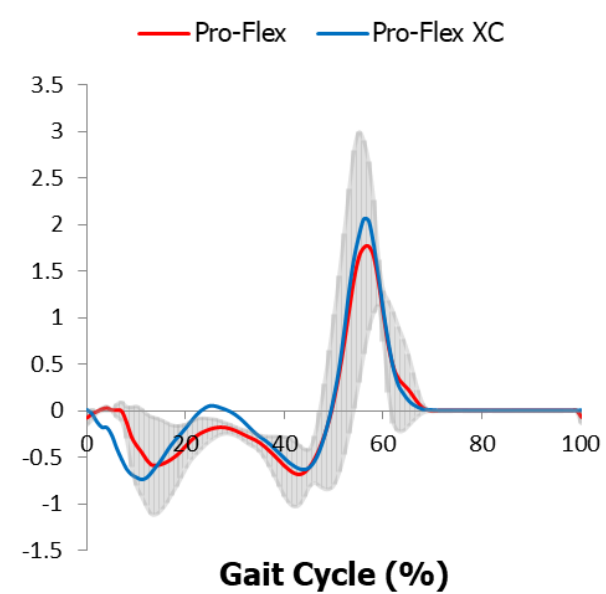

Figure 1. Mean prosthetic ankle angle and power during level, decline, and incline walking. Pro-Flex standard deviation in gray.

\section{ACKNOWLEDGE}

This study was financially supported by Mitacs and prosthetic components were provided by Össur. Courtney Bridgewater and Andrew Smith are acknowledged for their assistance with motion capture and data processing.

\section{REFERENCES}

1. Heitzmann, Daniel WW, et al. "Benefits of an increased prosthetic ankle range of motion for individuals with a transtibial amputation walking with a new prosthetic foot." Gait \& Posture, 64, 174-180, 2018. https://doi.org/10.1016/j.gaitpost.2018.06.022
2. Össur [Internet]. c2018 [cited 2018 Aug 14]. Pro-Flex. Available from: https://www.ossur.com/prosthetic- solutions/ products/dynamic-solutions/pro-flex.

3. E. H. Sinitski, E. D. Lemaire, and N. Baddour, "Evaluation of motion platform embedded with force plate-instrumented treadmill.” J. Rehabil. Res. Dev., 52(2), 221-233, 2015. doi: 10.1682/JRRD.2013.11.0244.

4. Össur [Internet]. c2018 [cited 2018 Aug 14]. Pro-Flex XC. Available from: https://www.ossur.com/prosthetic-solutions/ products/ dynamic-solutions/pro-flex-xc. 